\title{
Lie symmetry and approximate Hojman conserved quantity of Appell equations for a weakly nonholonomic system
}

\author{
Yuelin Han • Xiaoxiao Wang • Meiling Zhang • \\ Liqun Jia
}

Received: 19 May 2012 / Accepted: 14 June 2012 / Published online: 13 December 2012

(C) The Author(s) 2012. This article is published with open access at Springerlink.com

\begin{abstract}
For a weakly nonholonomic system, the Lie symmetry and approximate Hojman conserved quantity of Appell equations are studied. Based on the Appell equations for a weakly nonholonomic system under special infinitesimal transformations of a group in which the time is invariable, the definition of the Lie symmetry of the weakly nonholonomic system and its first-degree approximate holonomic system are given. With the aid of the structure equation that the gauge function satisfies, the exact and approximate Hojman conserved quantities deduced directly from the Lie symmetry are derived. Finally, an example is given to study the exact and approximate Hojman conserved quantity of the system.
\end{abstract}

Keywords Weakly nonholonomic system · Appell equations $\cdot$ Lie symmetry $\cdot$ Approximate Hojman conserved quantity

\section{Introduction}

In recent years, research on the dynamics of nonholonomic system has made great progress [1-7]. A special nonholonomic system whose constraint equations contain a small parameter is called a weakly

Y. Han $\cdot$ X. Wang $\cdot$ M. Zhang $\cdot$ L. Jia $(\bowtie)$

School of Science, Jiangnan University, Wuxi 214122,

People's Republic of China

e-mail: jlq0000@163.com nonholonomic system. This system becomes a holonomic system when the small parameter equals zero. Researchers have already discussed the equations of motion, approximate solution, canonical transformation, and stability of the weakly nonholonomic system [8-10]. A previous study [11] examined the special Mei symmetry and approximate conserved quantity of Appell equations for a weakly nonholonomic system. Appell equations are important in analytical mechanics and belong to one of the three types of mechanical systems in the theory of analytical mechanics. In the recent 20 years, Chinese researchers have gained fruitful achievements in research, promotion, and application of Appell equations [12-17]. Since 2000, Chinese researchers have made some achievements in this research area, especially in Lie symmetry [35-47] of constrained mechanical systems [18-34]. However, there are fewer results on Appell equations. To solve Appell equations, Mei Feng-Xiang first derived the Noether conserved quantity deduced indirectly from the Noether symmetry using Mei symmetry [48]. In the present study, we examined Lie symmetry and approximate Hojman conserved quantity for Appell equations of a weakly nonholonomic system. First, the Appell equations and their first-degree approximation formulas were established for a weakly nonholonomic system. Subsequently, the definition and criterion of Lie symmetry were obtained. Then, the exact and approximate Hojman conserved quantities were directly deduced from the Lie symmetry. Finally, an example 
has been given to illustrate the application of the theoretical results of this study.

\section{Differential equations of motion of a weakly nonholonomic system}

Suppose that the position of a mechanical system is determined by the $n$ generalized coordinates, $q_{s}(s=$ $1,2, \ldots, n)$, and whose motion is subject to the $g$ ideal bilateral homogeneous nonholonomic constraints of Chetaev type

$$
\begin{aligned}
& f_{\beta}=\dot{q}_{\varepsilon+\beta}-b B_{\varepsilon+\beta, \delta}\left(t, q_{s}\right) \dot{q}_{\delta}=0 \\
& \quad(\beta=1,2, \ldots, g ; \delta=1,2, \ldots, \varepsilon ; \varepsilon=n-g ; \\
& \quad s=1,2, \ldots, n),
\end{aligned}
$$

where $b$ is a small parameter. When $b=0$, the constraint equation (1) becomes a constraint equation of the holonomic system. The Chetaev condition of the constraint equation (1) imposed on the virtual displacement $\delta q_{s}$ is

$$
\frac{\partial f_{\beta}}{\partial \dot{q}_{s}} \delta q_{s}=0 .
$$

The Appell function of the system is $S=S(t, \boldsymbol{q}, \dot{\boldsymbol{q}}, \ddot{\boldsymbol{q}})$, and the generalized forces are $Q_{s}=Q_{s}(t, \boldsymbol{q}, \dot{\boldsymbol{q}})$; thus, the Appell equations of the system are

$$
\frac{\partial S}{\partial \ddot{q}_{s}}=Q_{s}+\lambda_{\beta} \frac{\partial f_{\beta}}{\partial \dot{q}_{s}},
$$

where $\lambda_{\beta}$ denotes the constraint multipliers in (3). If the system is nonsingular, one can solve all the constraint multipliers $\lambda_{\beta}(t, b, \boldsymbol{q}, \dot{\boldsymbol{q}})$ using (1)-(2). Equation (3) can also be expressed as

$$
\frac{\partial S}{\partial \ddot{q}_{s}}=Q_{s}+\Lambda_{s},
$$

where

$$
\Lambda_{s}=\Lambda_{s}(t, \boldsymbol{q}, \dot{\boldsymbol{q}})=\lambda_{\beta} \frac{\partial f_{\beta}}{\partial \dot{q}_{s}}
$$

are the generalized constraint forces. Equation (4) is the equation of the holonomic system, corresponding to those of the weakly nonholonomic system (1) and (3). It has been proved that the solution of the relevant holonomic system (4) corresponding to the weakly nonholonomic system (1) and (3) will give the motion if the initial conditions of motion satisfy the constraint equation (1).

To discuss the approximate solution of the weakly nonholonomic system, we can expand the generalized constraint forces $\Lambda_{s}$ as a power series

$$
\begin{aligned}
\Lambda_{s}= & \Lambda_{s 0}(t, \boldsymbol{q}, \dot{\boldsymbol{q}})+b \Lambda_{s 1}(t, \boldsymbol{q}, \dot{\boldsymbol{q}}) \\
& +b^{2} \Lambda_{s 2}(t, \boldsymbol{q}, \dot{\boldsymbol{q}})+\cdots .
\end{aligned}
$$

Then, the first-degree approximation of (4) can be expressed as

$\frac{\partial S}{\partial \ddot{q}_{s}}=Q_{s}+\Lambda_{s 0}+b \Lambda_{s 1}$.

Also, all generalized accelerations can be solved from (4) and can be written as

$\ddot{q}_{s}=\alpha_{S}(t, b, \boldsymbol{q}, \dot{\boldsymbol{q}})$.

From (7) we obtain

$\ddot{q}_{s}=\alpha_{s 0}(t, \boldsymbol{q}, \dot{\boldsymbol{q}})+b \alpha_{s 1}(t, \boldsymbol{q}, \dot{\boldsymbol{q}})$.

\section{Determining equation and definition of Lie symmetry}

Considering the Lie symmetry for (4) and (7), let us take the special infinitesimal transformations of group in which the time is invariable as

$t^{*}=t, \quad q_{s}^{*}\left(t^{*}\right)=q_{s}(t)+\Delta q_{s}, \quad(s=1, \ldots, n)$.

Equation (10) can also be extended into

$t^{*}=t, \quad q_{s}^{*}\left(t^{*}\right)=q_{s}(t)+\varepsilon \xi_{s}(t, \boldsymbol{q}, \dot{\boldsymbol{q}})$,

where $\varepsilon$ is an infinitesimal parameter, and $\xi_{s}$ denotes the generation functions of the infinitesimal transformations. By introducing the infinitesimal generator vector $X^{(0)}$ and its first and second extended infinitesimal generators $\tilde{X}^{(1)}$ and $\tilde{X}^{(2)}$, we get

$$
\begin{aligned}
X^{(0)} & =\xi_{s} \frac{\partial}{\partial q_{s}}, \\
\tilde{X}^{(1)} & =X^{(0)}+\frac{\overline{\mathrm{d}} \xi_{s}}{\mathrm{~d} t} \frac{\partial}{\partial \dot{q}_{s}}, \\
\tilde{X}^{(2)} & =\tilde{X}^{(1)}+\frac{\overline{\mathrm{d}}}{\mathrm{d} t} \frac{\overline{\mathrm{d}} \xi_{s}}{\mathrm{~d} t} \frac{\partial}{\partial \ddot{q}_{s}} .
\end{aligned}
$$


Since $\frac{\partial S}{\partial \ddot{q}_{s}}=\frac{\mathrm{d}}{\mathrm{d} t} \frac{\partial T}{\partial \dot{q}_{s}}-\frac{\partial T}{\partial q_{s}}$, the determining equation of Lie symmetry (4) can be written as

$\tilde{X}^{(2)}\left(\frac{\partial S}{\partial \ddot{q}_{S}}\right)=\tilde{X}^{(1)}\left(Q_{S}\right)+\tilde{X}^{(1)}\left(\Lambda_{S}\right)$.

Equation (8) can be written as

$\frac{\overline{\mathrm{d}}}{\mathrm{d} t} \frac{\overline{\mathrm{d}}}{\mathrm{d} t} \xi_{s}=\frac{\partial \alpha_{s}}{\partial q_{k}} \xi_{k}+\frac{\partial \alpha_{s}}{\partial \dot{q}_{k}} \frac{\overline{\mathrm{d}}}{\mathrm{d} t} \xi_{k}$.

Equation (7) can be obtained as

$\tilde{X}^{(2)}\left(\frac{\partial S}{\partial \ddot{q}_{s}}\right)=\tilde{X}^{(1)}\left(Q_{s}\right)+\tilde{X}^{(1)}\left(\Lambda_{s 0}\right)+\tilde{X}^{(1)}\left(b \Lambda_{s 1}\right)$,

and (9) can be rewritten as

$\frac{\overline{\mathrm{d}}}{\mathrm{d} t} \frac{\overline{\mathrm{d}}}{\mathrm{d} t} \xi_{s}=\frac{\partial\left(\alpha_{s 0}+b \alpha_{s 1}\right)}{\partial q_{k}} \xi_{k}+\frac{\partial\left(\alpha_{s 0}+b \alpha_{s 1}\right)}{\partial \dot{q}_{k}} \frac{\overline{\mathrm{d}}}{\mathrm{d} t} \xi_{k}$.

Definition 1 If the infinitesimal generator $\xi_{s}$ satisfies (13) or (14), then the relevant symmetry is Lie symmetry of the weakly nonholonomic system (1) and (3) or holonomic system (4) corresponding to the weakly nonholonomic system (1) and (3).

The restriction equation of Lie symmetry for the weakly nonholonomic constraints (1) under the special infinitesimal transformations (11) can be expressed as

$\tilde{X}^{(1)}\left\{f_{\beta}(t, b, \boldsymbol{q}, \dot{\boldsymbol{q}})\right\}=0$.

Definition 2 If the infinitesimal generator $\xi_{s}$ satisfies (13) or (14) and the restriction equation (17), then the relevant symmetry is weakly Lie symmetry of the weakly nonholonomic system (1) and (3) or holonomic system (4) corresponding to the weakly nonholonomic system (1) and (3).

Considering the Appell-Chetaev condition (2) imposed on the virtual displacement $\delta q_{s}$, we have the following additional restriction equation:

$\frac{\partial f_{\beta}}{\partial \dot{q}_{s}} \xi_{s}=0, \quad(\beta=1, \ldots, g ; s=1, \ldots, n)$.

Definition 3 If the infinitesimal generator $\xi_{s}$ satisfies (13) or (14), the restriction equation (17), and the additional restriction equation (18), then the relevant sym- metry is strong Lie symmetry of the weakly nonholonomic system (1) and (3) or holonomic system (4) corresponding to the weakly nonholonomic system (1) and (3).

Definition 4 If the infinitesimal generator $\xi_{s}$ satisfies (15) or (16), then the relevant symmetry is Lie symmetry of the first-degree approximate holonomic system (7) corresponding to the weakly nonholonomic system (1) and (3).

Definition 5 If the infinitesimal generator $\xi_{s}$ satisfies (15) or (16) and the restriction equation (17), then the relevant symmetry is weakly Lie symmetry of the firstdegree approximate holonomic system (7) corresponding to the weakly nonholonomic system (1) and (3).

Definition 6 If the infinitesimal generator $\xi_{s}$ satisfies (15) or (16), the restriction equation (17), and the additional restriction equation (18), then the relevant symmetry is strong Lie symmetry of the first-degree approximate holonomic system (7) corresponding to the weakly nonholonomic system (1) and (3).

\section{Hojman conserved quantity deduced from the special Lie symmetry}

Proposition 1 [49] If the infinitesimal generator $\xi_{s}$ satisfies the determining equation (13) or (14) and if the function $\mu=\mu(t, q, \dot{q})$ satisfies the equation

$\frac{\partial \alpha_{s}}{\partial \dot{q}_{s}}+\frac{\overline{\mathrm{d}}}{\mathrm{d} t} \ln \mu=0$,

then the exact Hojman conserved quantity can be deduced from the Lie symmetry of Appell equations for the weakly nonholonomic system (1) and (3), or the corresponding holonomic system (4) is

$I_{H}=\frac{1}{\mu} \frac{\partial}{\partial q_{s}}\left(\mu \xi_{s}\right)+\frac{1}{\mu} \frac{\partial}{\partial \dot{q}_{s}}\left(\mu \frac{\overline{\mathrm{d}}}{\mathrm{d} t} \xi_{s}\right)=$ const.

Proposition 2 If the infinitesimal generator $\xi_{s}$ satisfies (13) or (14) and the restriction equation (17), and if the function $\mu=\mu(t, \boldsymbol{q}, \dot{\boldsymbol{q}})$ satisfies (19), then the exact Hojman conserved quantity (20) can be deduced from weakly Lie symmetry of Appell equations for the weakly nonholonomic system (1) and (3), or the corresponding holonomic system (4). 
Proposition 3 If the infinitesimal generator $\xi_{s}$ satisfies (13) or (14), the restriction equation (17), and the additional restriction equation (18), and if the function $\mu=\mu(t, \boldsymbol{q}, \dot{\boldsymbol{q}})$ satisfies (19), then the exact Hojman conserved quantity (20) can be deduced from strong Lie symmetry of Appell equations for the weakly nonholonomic system (1) and (3) or the corresponding holonomic system (4).

Proposition 4 If the infinitesimal generator $\xi_{s}$ satisfies (15) or (16), and if a function $\mu=\mu(t, \boldsymbol{q}, \dot{\boldsymbol{q}})$ satisfies

$$
\frac{\partial\left(\alpha_{s 0}+b \alpha_{s 1}\right)}{\partial \dot{q}_{s}}+\frac{\overline{\mathrm{d}}}{\mathrm{d} t} \ln \mu=0,
$$

then the approximate Hojman conserved quantity (20) can be deduced from Lie symmetry of Appell equations for the first-degree approximate holonomic system (7).

Proof Taking the derivative of Eq. (20) with respect to $t$, we can obtain

$$
\begin{aligned}
\frac{\overline{\mathrm{d}}}{\mathrm{d} t} I_{H}= & \frac{\overline{\mathrm{d}}}{\mathrm{d} t}\left(\frac{1}{\mu} \frac{\partial \mu}{\partial q_{s}} \xi_{s}\right)+\frac{\overline{\mathrm{d}}}{\mathrm{d} t} \frac{\partial \xi_{s}}{\partial q_{s}}+\frac{\overline{\mathrm{d}}}{\mathrm{d} t}\left[\frac{1}{\mu} \frac{\partial \mu}{\partial \dot{q}_{s}} \frac{\overline{\mathrm{d}}}{\mathrm{d} t} \xi_{s}\right. \\
& \left.+\frac{\partial}{\partial \dot{q}_{s}} \frac{\overline{\mathrm{d}}}{\mathrm{d} t} \xi_{s}\right] .
\end{aligned}
$$

Furthermore, we can easily prove that

$$
\begin{aligned}
& \frac{\overline{\mathrm{d}}}{\mathrm{d} t} \frac{\partial}{\partial \dot{q}_{s}} \frac{\overline{\mathrm{d}}}{\mathrm{d} t} \xi_{s}= \frac{\partial}{\partial \dot{q}_{s}} \frac{\overline{\mathrm{d}}}{\mathrm{d} t}\left(\frac{\overline{\mathrm{d}}}{\mathrm{d} t} \xi_{s}\right)-\frac{\partial}{\partial q_{s}} \frac{\overline{\mathrm{d}}}{\mathrm{d} t} \xi_{s} \\
&-\frac{\partial}{\partial \dot{q}_{k}}\left(\frac{\overline{\mathrm{d}}}{\mathrm{d} t} \xi_{s}\right), \\
& \frac{\overline{\mathrm{d}}}{\mathrm{d} t} \frac{\partial \xi_{s}}{\partial q_{s}}=\frac{\partial}{\partial q_{s}} \frac{\overline{\mathrm{d}}}{\mathrm{d} t} \xi_{s}-\frac{\partial\left(\alpha_{k 0}+b \alpha_{k 1}\right)}{\partial q_{s}} \frac{\partial \xi_{s}}{\partial \dot{q}_{k}} .
\end{aligned}
$$

By taking the partial derivative of (16) with respect to $\dot{q}_{s}$ and the summation of terms with subscript $s$, we have

$$
\begin{aligned}
\frac{\partial}{\partial \dot{q}_{s}} \frac{\overline{\mathrm{d}}}{\mathrm{d} t}\left(\frac{\overline{\mathrm{d}}}{\mathrm{d} t} \xi_{s}\right)= & \frac{\partial}{\partial \dot{q}_{s}}\left(\frac{\partial\left(\alpha_{s 0}+b \alpha_{s 1}\right)}{\partial q_{k}} \xi_{k}\right) \\
& +\frac{\partial}{\partial \dot{q}_{s}}\left(\frac{\partial\left(\alpha_{s 0}+b \alpha_{s 1}\right)}{\partial \dot{q}_{k}} \frac{\overline{\mathrm{d}}}{\mathrm{d} t} \xi_{k}\right) .
\end{aligned}
$$

By substituting (23) and (24) into (22), we have

$$
\frac{\overline{\mathrm{d}}}{\mathrm{d} t} I_{H}=\frac{\overline{\mathrm{d}}}{\mathrm{d} t}\left(\frac{1}{\mu} \frac{\partial \mu}{\partial q_{s}} \xi_{s}\right)+\frac{\overline{\mathrm{d}}}{\mathrm{d} t}\left(\frac{1}{\mu} \frac{\partial \mu}{\partial \dot{q}_{s}} \frac{\overline{\mathrm{d}}}{\mathrm{d} t} \xi_{s}\right)
$$

$$
+\frac{\partial^{2}\left(\alpha_{s 0}+b \alpha_{s 1}\right)}{\partial q_{k} \partial \dot{q}_{s}} \xi_{k}+\frac{\partial^{2}\left(\alpha_{s 0}+b \alpha_{s 1}\right)}{\partial \dot{q}_{k} \partial \dot{q}_{s}} \frac{\overline{\mathrm{d}}}{\mathrm{d} t} \xi_{k} .
$$

By using (21) we obtain

$$
\begin{aligned}
\frac{\overline{\mathrm{d}}}{\mathrm{d} t}\left(\frac{1}{\mu} \frac{\partial \mu}{\partial q_{s}} \xi_{s}\right)= & -\frac{\partial^{2}\left(\alpha_{s 0}+b \alpha_{s 1}\right)}{\partial q_{k} \partial \dot{q}_{s}} \xi_{k}+\frac{1}{\mu} \frac{\partial \mu}{\partial q_{s}} \frac{\overline{\mathrm{d}}}{\mathrm{d} t} \xi_{s} \\
& -\frac{1}{\mu} \frac{\partial \mu}{\partial \dot{q}_{k}} \frac{\partial\left(\alpha_{k 0}+b \alpha_{k 1}\right)}{\partial q_{s}} \xi_{s}
\end{aligned}
$$

$$
\begin{aligned}
\frac{\overline{\mathrm{d}}}{\mathrm{d} t}\left(\frac{1}{\mu} \frac{\partial \mu}{\partial \dot{q}_{s}} \frac{\overline{\mathrm{d}}}{\mathrm{d} t} \xi_{s}\right) \\
=-\frac{\partial^{2}\left(\alpha_{s 0}+b \alpha_{s 1}\right)}{\partial \dot{q}_{k} \partial \dot{q}_{s}} \frac{\overline{\mathrm{d}}}{\mathrm{d} t} \xi_{k} \\
\quad-\frac{1}{\mu} \frac{\partial\left(\alpha_{k 0}+b \alpha_{k 1}\right)}{\partial \dot{q}_{s}} \frac{\partial \mu}{\partial \dot{q}_{k}} \frac{\overline{\mathrm{d}}}{\mathrm{d} t} \xi_{s} \\
\quad+\frac{1}{\mu} \frac{\partial \mu}{\partial \dot{q}_{s}} \frac{\overline{\mathrm{d}}}{\mathrm{d} t}\left(\frac{\overline{\mathrm{d}}}{\mathrm{d} t} \xi_{s}\right)-\frac{1}{\mu} \frac{\partial \mu}{\partial q_{s}} \frac{\overline{\mathrm{d}}}{\mathrm{d} t} \xi_{s} .
\end{aligned}
$$

By substituting (26) and (27) into (25) we have

$$
\begin{aligned}
\frac{\overline{\mathrm{d}}}{\mathrm{d} t} I_{H}= & \frac{1}{\mu} \frac{\partial \mu}{\partial \dot{q}_{s}}\left\{\frac{\overline{\mathrm{d}}}{\mathrm{d} t}\left(\frac{\overline{\mathrm{d}}}{\mathrm{d} t} \xi_{s}\right)-\frac{\partial\left(\alpha_{s 0}+b \alpha_{s 1}\right)}{\partial \dot{q}_{k}} \frac{\overline{\mathrm{d}}}{\mathrm{d} t} \xi_{k}\right. \\
& \left.-\frac{\partial\left(\alpha_{s 0}+b \alpha_{s 1}\right)}{\partial q_{k}} \xi_{k}\right\}=0 .
\end{aligned}
$$

Thus, our proof is completed.

Proposition 5 If the infinitesimal generator $\xi_{s}$ satisfies (15) or (16) and the restriction equation (17), and if the function $\mu=\mu(t, \boldsymbol{q}, \dot{\boldsymbol{q}})$ satisfies (21), then the approximate Hojman conserved quantity (20) can be deduced from weakly Lie symmetry of Appell equations for the first-degree approximate holonomic system (7).

Proposition 6 If the infinitesimal generator $\xi_{\text {s }}$ satisfies (15) or (16), the restriction equation (17), and the additional restriction equation (18), and if the function $\mu=\mu(t, \boldsymbol{q}, \dot{\boldsymbol{q}})$ satisfies $(21)$, then the approximate Hojman conserved quantity (20) can be deduced from strong Lie symmetry of Appell equations for the firstdegree approximate holonomic system (7).

\section{An illustrative example}

In the following, we have given an example to illustrate the application of the above-mentioned results. 
Let us consider a weakly nonholonomic system where a particle of unit quality moves in the twodimensional space that is perpendicular to the Earth's surface and whose Appell function, generalized force, and constraint equations are

$S=\frac{1}{2}\left(\ddot{q}_{1}^{2}+\ddot{q}_{2}^{2}\right)$,

$Q_{1}=0, \quad Q_{2}=-g$,

$f=\dot{q}_{2}-b t \dot{q}_{1}=0$.

Let us now study the Lie symmetry and Hojman conserved quantity of the system.

By substituting (28)-(30) into (3) we can get

$\ddot{q}_{1}=-\lambda b t, \quad \ddot{q}_{2}=-g+\lambda$.

From (30) and (31) we obtain

$\lambda=\frac{b \dot{q}_{1}+g}{1+b^{2} t^{2}}$.

Thus, we have

$\ddot{q}_{1}=-\frac{b^{2} t \dot{q}_{1}+b t g}{1+b^{2} t^{2}}, \quad \ddot{q}_{2}=\frac{b \dot{q}_{1}-b^{2} t^{2} g}{1+b^{2} t^{2}}$.

According to the determining equation (14) of Lie symmetry, we can obtain

$\ddot{\xi}_{1}=-\frac{b^{2} t}{1+b^{2} t^{2}} \dot{\xi}_{1}$,

$\ddot{\xi}_{2}=\frac{b}{1+b^{2} t^{2}} \dot{\xi}_{1}$.

The above-mentioned equations (34) have the following solutions:

$\xi_{1}=\xi_{2}=1$,

$\xi_{1}=1, \quad \xi_{2}=\left(\dot{q}_{1}+b t \dot{q}_{2}-b q_{2}+\frac{1}{2} g b t^{2}\right)^{2}$.

The restriction equation (17) gives

$\dot{\xi}_{2}-b t \dot{\xi}_{1}=0$.

From the additional restriction equation (18) we get

$\xi_{2}-b t \xi_{1}=0$.

Apparently, the infinitesimal generators (35) and (36) satisfy the restriction equation (37) but do not satisfy the additional restriction equation (38), respectively. Therefore, they are the respective infinitesimal generators of Lie symmetry and weakly Lie symmetry for the weakly nonholonomic system.

Furthermore, from (18) we obtain

$-\frac{b^{2} t}{1+b^{2} t^{2}}+\frac{\overline{\mathrm{d}}}{\mathrm{d} t} \ln \mu=0$.

The above-mentioned equation (39) has the following solutions:

$\mu=\sqrt{1+b^{2} t^{2}}$,

$\mu=\sqrt{1+b^{2} t^{2}}\left(\dot{q}_{1}+b t \dot{q}_{2}-b q_{2}+\frac{1}{2} g b t^{2}\right)$.

From (35), (40), and (20) we obtain

$I_{H_{1}}=0=$ const.

From (35), (41), and (20) we get

$I_{H_{2}}=-b\left(\dot{q}_{1}+b t \dot{q}_{2}-b q_{2}+\frac{1}{2} g b t^{2}\right)^{-1}=$ const.

From (36), (40), and (20) we obtain

$I_{H_{3}}=-2 b\left(\dot{q}_{1}+b t \dot{q}_{2}-b q_{2}+\frac{1}{2} g b t^{2}\right)=$ const.

Equations (36), (41), and (20) give

$I_{H_{4}}=-3 b\left(\dot{q}_{1}+b t \dot{q}_{2}-b q_{2}+\frac{1}{2} g b t^{2}\right)=$ const.

Based on Propositions 1 and 2, we know that $I_{H_{1}}$, $I_{H_{2}}, I_{H_{3}}$, and $I_{H_{4}}$ are the exact Hojman conserved quantity of Lie symmetry and weakly Lie symmetry for the weakly nonholonomic system (31) or the corresponding holonomic system (33), where $I_{H_{1}}$ is the common conserved quantity. As the infinitesimal generators (35) and (36) do not satisfy the additional restriction equation (38), from Proposition 3 we know that these conserved quantities are not the exact Hojman conserved quantity of strong Lie symmetry for the weakly nonholonomic system (31) or the corresponding holonomic system (33).

The first-degree approximate equations of (33) are

$\ddot{q}_{1}=-b \operatorname{tg}, \quad \ddot{q}_{2}=b \dot{q}_{1}$. 
The determining equation (16) of Lie symmetry gives $\ddot{\xi}_{1}=0$,

$\ddot{\xi}_{2}=b \dot{\xi}_{1}$.

The above-mentioned equations (47) have the following solutions:

$\xi_{1}=\xi_{2}=1$,

$\xi_{1}=\left(\dot{q}_{2}-b q_{1}\right)^{2}, \quad \xi_{2}=0$,

$\xi_{1}=\left(\dot{q}_{1}+\dot{q}_{2}-b q_{1}+\frac{1}{2} g b t^{2}\right)^{2}, \quad \xi_{2}=0$.

From the restriction equation (17) we get

$\dot{\xi}_{2}-b t \dot{\xi}_{1}=0$.

Furthermore, from the additional restriction equation (18) we get

$\xi_{2}-b t \xi_{1}=0$.

The infinitesimal generators (48)-(50) satisfy the restriction equation (51) but do not satisfy the additional restriction equation (52). Therefore, they can be considered as the infinitesimal generators of Lie symmetry and weakly Lie symmetry for the first-degree approximate holonomic system.

From (21) we obtain

$\frac{\overline{\mathrm{d}}}{\mathrm{d} t} \ln \mu=0$.

The above-mentioned equation (53) has the following solutions:

$\mu=1$,

$\mu=\dot{q}_{2}-b q_{1}$,

$\mu=\dot{q}_{1}+\dot{q}_{2}-b q_{1}+\frac{1}{2} g b t^{2}$.

Therefore, from (48), (54), and (20) we obtain

$I_{H_{5}}=0$.

From (48), (55), and (20) we get

$I_{H_{6}}=-b\left(\dot{q}_{2}-b q_{1}\right)^{-1}=$ const.

From (48), (56), and (20) we can obtain

$I_{H_{7}}=-2 b\left(\dot{q}_{1}+\dot{q}_{2}-b q_{1}+\frac{1}{2} g b t^{2}\right)^{-1}=$ const.
Equations (49), (54), and (20) give

$I_{H_{8}}=-2 b\left(\dot{q}_{2}-b q_{1}\right)=$ const.

From (49), (55), and (20) we get

$I_{H_{9}}=-3 b\left(\dot{q}_{2}-b q_{1}\right)=$ const.

From (50), (54), and (20) we obtain

$I_{H_{10}}=-2 b\left(\dot{q}_{1}+\dot{q}_{2}-b q_{1}+\frac{1}{2} g b t^{2}\right)=$ const.

From (50), (56), and (20) we can acquire

$I_{H_{11}}=-3 b\left(\dot{q}_{1}+\dot{q}_{2}-b q_{1}+\frac{1}{2} g b t^{2}\right)=$ const.

According Propositions 4 and 5, we know that $I_{H_{5}}$, $I_{H_{6}}, I_{H_{7}}, I_{H_{8}}, I_{H_{9}}, I_{H_{10}}$, and $I_{H_{11}}$ are the approximate Hojman conserved quantities of Lie symmetry and weakly Lie symmetry for the first-degree approximate system (46), where $I_{H_{5}}$ is the common conserved quantity. As the infinitesimal generators (48)(50) do not satisfy the additional restriction equation (52), from Proposition 6 we know that these conserved quantities are not the approximate Hojman conserved quantities of strong Lie symmetry for the first-degree approximate system (46).

By taking the derivatives of expressions (57)-(63) with respect to time $t$, according to (31), we respectively obtain

$\dot{I}_{H_{5}}=0$,

$\dot{I}_{H_{6}}=-b \frac{\ddot{q}_{2}-b \dot{q}_{1}}{\left(\dot{q}_{2}-b q_{1}\right)^{2}}=\frac{b^{4} t^{2} \dot{q}_{1}+b^{3} t^{2} g}{\left(1+b^{2} t^{2}\right)\left(\dot{q}_{2}-b q_{1}\right)^{2}}$

$=\mathrm{O}\left(b^{3}\right)$,

$\dot{I}_{H_{7}}=-2 b \frac{\ddot{q}_{1}+\ddot{q}_{2}-b \dot{q}_{1}+g b t}{\left(\dot{q}_{1}+\dot{q}_{2}-b q_{1}+\frac{1}{2} g b t^{2}\right)^{2}}$

$=\frac{2\left(b^{3} t \dot{q}_{1}+b^{4} t^{2} \dot{q}_{1}+b^{3} t^{2} g-b^{4} t^{3} g\right)}{\left(1+b^{2} t^{2}\right)\left(\dot{q}_{1}+\dot{q}_{2}-b q_{1}+\frac{1}{2} g b t^{2}\right)^{2}}$

$=\mathrm{O}\left(b^{3}\right)$,

$\dot{I}_{H_{8}}=-2 b\left(\ddot{q}_{2}-b \dot{q}_{1}\right)=\frac{2\left(b^{4} t^{2} \dot{q}_{1}+g b^{3} t^{2}\right)}{1+b^{2} t^{2}}$

$=\mathrm{O}\left(b^{3}\right)$ 


$$
\begin{aligned}
\dot{I}_{H_{9}} & =-3 b\left(\ddot{q}_{2}-b \dot{q}_{1}\right)=\frac{3\left(b^{4} t^{2} \dot{q}_{1}+g b^{3} t^{2}\right)}{1+b^{2} t^{2}} \\
& =\mathrm{O}\left(b^{3}\right), \\
\dot{I}_{H_{10}} & =-2 b\left(\ddot{q}_{1}+\ddot{q}_{2}-b \dot{q}_{1}+g b t\right) \\
& =\frac{2\left(b^{3} t \dot{q}_{1}+b^{4} t^{2} \dot{q}_{1}+b^{3} t^{2} g-b^{4} t^{3} g\right)}{\left(1+b^{2} t^{2}\right)} \\
& =\mathrm{O}\left(b^{3}\right), \quad \\
\dot{I}_{H_{11}} & =-3 b\left(\ddot{q}_{1}+\ddot{q}_{2}-b \dot{q}_{1}-g b t\right) \\
& =\frac{3\left(b^{3} t \dot{q}_{1}+b^{4} t^{2} \dot{q}_{1}+b^{3} t^{2} g-b^{4} t^{3} g\right)}{\left(1+b^{2} t^{2}\right)} \\
& =\mathrm{O}\left(b^{3}\right) .
\end{aligned}
$$

Thus, the above-mentioned quantities are the approximate Hojman conserved quantities of the weakly nonholonomic system.

\section{Conclusions}

This study examined the theory of Lie symmetry and Hojman conserved quantity of Appell equations for a weakly nonholonomic system, and analyzed the exact and approximate Hojman conserved quantities. The theoretical results apply not only to the weakly nonholonomic system, when the small parameter $b$ is zero, but also to the holonomic system. The method through which the approximate conserved quantity can be obtained by expanding the generalized constraint force $\Lambda_{s}$ as a power series in small parameter $b$ can also be applied to other mechanical and physical systems with other small parameters. Thus, the results of this study have a great significance in improving and developing Lie symmetry and conserved quantity of mechanical system.

Acknowledgements Project supported by the National Natural Science Foundation of China (Grant Nos. 11142014 and 61178032) and Project supported by scientific research and innovation plan for College Graduates of Jiangsu province (Grant No. CXLX12_0720)

Open Access This article is distributed under the terms of the Creative Commons Attribution License which permits any use, distribution, and reproduction in any medium, provided the original author(s) and the source are credited.

\section{References}

1. Neimark, J.I., Fufaev, N.A.: Dynamics of Nonholonomic Systems. AMS, Providence (1972)

2. Mei, F.X.: Foundations of Mechanics of Nonholonomic Systems. Beijing Institute of Technology Press, Beijing (1985)

3. Bloch, A.M., Krishnaprasad, P.S., Marsden, J.E., Murray, R.M.: Arch. Ration. Mech. Anal. 136, 21 (1996)

4. Ostrovskaya, S., Angels, J.: Nonholonomic systems revisited within the frame work of analytical mechanics. Appl. Mech. Rev. 51, 415-433 (1998)

5. Mei, F.X.: Nonholonomic mechanics. Appl. Mech. Rev. 53, 283-305 (2000)

6. Guo, Y.X., Luo, S.K., Mei, F.X.: Progress of geometric dynamics of non-holonomic constrained mechanical systems: Lagrange theory and others. Adv. Mech. 34(4), 477-492 (2004)

7. Zegzhda, S.A., Soltakhanov, S.K., Yushkov, M.P.: Equations of Motion of Nonholonomic Systems and Variational Principles of Mechanics. New kind of Control Problems. FIMATLIT, Moscow (2005)

8. Mei, F.X.: Equations of motion for weak nonholonomic systems and their approximate solution. J. Beijing Inst. Technol. 9(3), 10-17 (1989)

9. Mei, F.X.: Canonical transformation of weak nonholonomic systems. Chin. Sci. Bull. 37, 1180-1183 (1992)

10. Mei, F.X.: On the stability of one type of weakly nonholonomic systems. J. Beijing Inst. Technol. 15, 237-242 (1995)

11. Jia, L.Q., Wang, X.X., Zhang, M.L., Han, Y.L.: Special Mei symmetry and approximate conserved quantity of Appell equations for a weakly nonholonomic system. Nonlinear Dyn. 69(4), 1807-1812 (2012)

12. Xue, W.X.: The generalization of Appell equation and Tzénoff equation. Acta Mech. Sin. 19(2), 156-164 (1987)

13. Luo, S.K.: Appell equations and form invariance of rotational relativistic systems. Acta Phys. Sin. 51(4), 712-717 (2002)

14. Cui, J.C., Zhang, Y.Y., Yang, X.F., Jia, L.Q.: Mei symmetry and Mei conserved quantity of Appell equations for a variable mass holonomic system. Chin. Phys. B 19(3), 030304 (2010)

15. Li, Y.C., Xia, L.L., Wang, X.M., Liu, X.W.: Lie-Mei symmetry and conserved quantities of Appell equation for a holonomic mechanical system. Acta Phys. Sin. 59(6), 3639-3642 (2010)

16. Jia, L.Q., Xie, Y.L., Zhang, Y.Y., Cui, J.C., Yang, X.F.: A new type of conserved quantity induced by Mei symmetry of Appell equation. Acta Phys. Sin. 59(11), 7552-7555 (2010)

17. Yang, X.F., Sun, X.T., Wang, X.X., Zhang, M.L., Jia, L.Q.: Mei symmetry and Mei conserved quantity of Appell equations for nonholonomic systems of Chetaev's type with variable mass. Acta Phys. Sin. 60(11), 111101 (2011)

18. Mei, F.X.: Applications of Lie Groups and Lie Algebras to Constrained Mechanical Systems. Science Press, Beijing (1999)

19. Mei, F.X., Chen, X.W.: Perturbation to the symmetries and adiabatic invariants of holonomic variable mass systems. Chin. Phys. 9(10), 721-725 (2000) 
20. Luo, S.K.: A new type of Lie symmetrical non-Noether conserved quantity for nonholonomic systems. Chin. Phys. 13(12), 2182-2186 (2004)

21. Cai, J.L.: Conformal invariance and conserved quantities of general holonomic systems. Chin. Phys. Lett. 25(5), 15231526 (2008)

22. Cai, J.L., Mei, F.X.: Conformal invariance and conserved quantity of Lagrange systems under Lie point transformation. Acta Phys. Sin. 57(9), 5369-5373 (2008)

23. Cai, J.L., Luo, S.K., Mei, F.X.: Conformal invariance and conserved quantity of Hamilton systems. Chin. Phys. B 17(9), 3170-3174 (2008)

24. Zhang, Y., Mei, F.X.: Effects of constraints on Noether symmetries and conserved quantities in a Birkhoffian system. Acta Phys. Sin. 53(8), 2419-2423 (2004)

25. Luo, S.K., Zhang, Y.F.: Advances in the Study of Dynamics of Constrained Systems. Science Press, Beijing (2008)

26. Fang, J.H.: A kind of conserved quantity of Mei symmetry for Lagrange system. Acta Phys. Sin. 58(6), 3617-3619 (2009)

27. Cai, J.L.: Conformal invariance and conserved quantities of Mei symmetry for general holonomic systems. Acta Phys. Sin. 58(1), 22-27 (2009)

28. Cai, J.L.: Conformal invariance and conserved quantities of Mei symmetry for Lagrange systems. Acta Phys. Pol. A 115(5), 854-856 (2009)

29. Xie, Y.L., Jia, L.Q.: Special Lie-Mei symmetry and conserved quantity of Appell equations expressed by Appell function. Chin. Phys. Lett. 27(12), 120201 (2010)

30. Zheng, S.W., Xie, J.F., Chen, X.W.: Another kind of conserved quantity induced directly from Mei symmetry of Tzénoff equations for holonomic systems. Acta Phys. Sin. 59(8), 5209-5212 (2010)

31. Jia, L.Q., Sun, X.T., Zhang, M.L., Wang, X.X., Xie, Y.L.: A type of new conserved quantity of Mei symmetry for Nielsen equations. Acta Phys. Sin. 60(8), 084501 (2011)

32. Jiang, W.A., Luo, S.K.: Mei symmetry leading to Mei conserved quantity of generalized Hamilton systems. Acta Phys. Sin. 60(6), 060201 (2011)

33. Jiang, W.A., Li, Z.J., Luo, S.K.: Mei symmetries and Mei conserved quantities for higher-order nonholonomic systems. Chin. Phys. B 20(3), 030202 (2011)

34. Cai, J.L.: Conformal invariance of Mei symmetry for the nonholonomic system of non-Chetaev's type. Nonlinear Dyn. 69(1-2), 487-493 (2012)

35. Mei, F.X.: Lie symmetries and conserved quantities of nonholonomic systems with servoconstraints. Acta Phys. Sin. 49(7), 1207-1210 (2000)
36. Zhang, Y., Xue, Y.: Lie symmetries of constrained Hamiltonian system with the second type of constraint. Acta Phys. Sin. 50(5), 816-819 (2001)

37. Zhang, H.B.: Lie symmetries and conserved quantities of non-holonomic mechanical systems with unilateral Vacco constraints. Chin. Phys. 11(1), 1-4 (2002)

38. Fang, J.H., Zhang, P.Y.: The conserved quantity of Hojman for mechanical systems with variable mass in phase space. Acta Phys. Sin. 53(12), 4041-4044 (2004)

39. Chen, X.W., Li, Y.M., Zhao, Y.H.: Lie symmetries, perturbation to symmetries and adiabatic invariants of Lagrange system. Phys. Lett. A 337(4-6), 274-278 (2005)

40. Xu, X.J., Mei, F.X., Zhang, Y.F.: Lie symmetry and conserved quantity of a system of first-order differential equations. Chin. Phys. 15(1), 19-21 (2006)

41. Chen, X.W., Liu, C.M., Li, Y.M.: Lie symmetries, perturbation to symmetries and adiabatic invariants of Poincaré equations. Chin. Phys. B 15(3), 470-474 (2006)

42. Luo, S.K., Guo, Y.X.: Lie symmetrical perturbation and adiabatic invariants of generalized Hojman type of relativistic Birkhoffian systems. Commun. Theor. Phys. 47, 25-30 (2007)

43. Luo, S.K.: A new type of non-Noether adiabatic invariants, i.e. adiabatic invariants of Lutzky type, for Lagrangian systems. Acta Phys. Sin. 56(10), 5580-5584 (2007)

44. Luo, S.K.: A new type of non-Noether adiabatic invariants for disturbed Lagrangian systems: adiabatic invariants of Generalized Lutzky type. Chin. Phys. Lett. 24(9), 24632466 (2007)

45. Li, Z.J., Jiang, W.A., Luo, S.K.: Lie symmetries, symmetrical perturbation and a new adiabatic invariant for disturbed nonholonomic systems. Nonlinear Dyn. 67(1), 445455 (2012)

46. Jiang, W.A., Luo, S.K.: A new type of non-Noether exact invariants and adiabatic invariants of generalized Hamiltonian systems. Nonlinear Dyn. 67(1), 475-482 (2012)

47. Jiang, W.A., Li, L., Li, Z.J., Luo, S.K.: Lie symmetrical perturbation and adiabatic invariants of non-Noether type for generalized Birkhoffian systems. Nonlinear Dyn. 67(2), 1075-1081 (2012)

48. Mei, F.X.: Form invariance of Appell equations. Chin. Phys. 10(3), 177-180 (2001)

49. Mei, F.X.: Symmetries and Conserved Quantities of Constrained Mechanical Systems. Beijing Institute of Technology Press, Beijing (2004) 